\title{
High SPRR1A expression is associated with poor survival in patients with colon cancer
}

\author{
YU DENG $^{1 *}$, XIN ZHENG $^{2 *}$, YIYI ZHANG ${ }^{1 *}$, MEIFANG XU ${ }^{2}$, CHENGWEI YE ${ }^{1}$, \\ MENGXIN LIN ${ }^{3}$, JIE PAN ${ }^{4}$, ZONGBIN XU $^{1}$, XINGRONG LU $^{1}$ and PAN CHI ${ }^{1}$ \\ Departments of ${ }^{1}$ Colorectal Surgery, ${ }^{2}$ Pathology, ${ }^{3}$ Oncology and ${ }^{4}$ Emergency Surgery, \\ Fujian Medical University Union Hospital, Fuzhou, Fujian 350001, P.R. China
}

Received March 30, 2019; Accepted November 7, 2019

DOI: $10.3892 / \mathrm{ol} .2020 .11453$

\begin{abstract}
High expression of small proline-rich protein 1A (SPRR1A) has been shown to be associated with tumor prognosis; however, the association between SPRR1A expression and colon cancer prognosis remains unclear. The present study sought to evaluate the association between SPRR1A expression and the clinicopathological characteristics of colon cancer, and to examine its potential prognostic value. A total of 114 patients with colon cancer were included. SPRR1A expression was evaluated by immunohistochemical staining, and the association between SPRR1A expression and clinicopathological parameters was analyzed. The prognostic value of SPRR1A was analyzed by Cox regression analysis, the Oncomine database and the R2 platform. SPRR1A expression was significantly increased in cancerous tissues compared with that in adjacent non-cancerous tissues. SPPRR1A expression was significantly associated with lymph node invasion. High SPRR1A expression was significantly associated with worse overall and disease-free survival rate. Cox regression analysis revealed that $\mathrm{T}$ stage, pathological $\mathrm{N}$ stage and high SPRR1A expression remained independent predictors for overall survival rate. The Oncomine database analysis demonstrated that SPRR1A mRNA expression levels were significantly increased in colorectal cancer tissues compared with those in adjacent non-cancerous tissues, and high SPRR1A expression was associated with a significantly worse event- and relapse-free survival time in the R2 platform. The data indicate that SPRR1A may serve as a potential biomarker for the prognosis of colon cancer.
\end{abstract}

Correspondence to: Professor Xingrong Lu or Dr Zongbin Xu, Department of Colorectal Surgery, Fujian Medical University Union Hospital, 29 Xinquan Road, Fuzhou, Fujian 350001, P.R. China

E-mail: fjxhlxr@163.com

E-mail: fjxhxzb@163.com

${ }^{*}$ Contributed equally

Key words: colon cancer, small proline-rich protein 1A, prognosis, biomarker

\section{Introduction}

In 2018 , the incidence rate of colon cancer was reported as 6.1, and 5.8\% mortality rate worldwide (1). Despite recent advances in radical surgery, adjuvant chemotherapy and target therapy, tumor recurrence and metastasis remain a major concern, and contribute to impaired survival in patients with colon cancer (2). The complexity of cancer makes it difficult to predict the prognosis for colon cancer $(3,4)$. Therefore, identifying prognostic biomarkers is warranted to improve the direction of treatment strategies and survival (5).

Small proline-rich protein 1A (SPRR1A), a member of the SPRR family, is a cross-linked envelope protein of keratinocytes $(6,7)$. Previously, it has been described as a specific marker of differentiation of keratinocytes and squamous epithelial cells $(6,8)$. Accumulating evidence has revealed the prognostic importance of the high expression of SPRR1A in various types of cancer, including diffuse large B-cell lymphomas (9), head and neck squamous cell carcinoma (10), and breast cancer (11). In addition, high SPRR1A expression was also reported in a murine preneoplastic intestine and in the normal intestinal mucosa of patients with colorectal cancer (CRC) (12). Colonic carcinogenesis is a multistage process involving environmental and genetic changes (13). Therefore, the aim of the present study was to examine whether high expression of SPRR1A may serve as a promising biomarker for colon cancer prognosis assessment. To the best of our knowledge, few studies have focused on this issue $(9,11,12)$.

In order to address this literature gap, the present study aimed to examine SPRR1A expression in cancerous and adjacent non-cancerous colon tissues using immunohistochemical staining. The associations between SPRR1A expression and clinicopathological parameters of colon cancer were subsequently investigated. The prognostic value of SPRR1A expression was examined using Cox regression and bioinformatics analysis.

\section{Materials and methods}

Patient tissue samples. Patients with colon cancer who underwent radical surgery between June 2010 and December 2010 at Fujian Medical University Union Hospital were identified from the prospectively maintained database. This study was 
approved by the Medical Ethics Committee of Fujian Medical University Union Hospital and all patients provided written informed consent on admission. Inclusion criteria included pathologically proven colon adenocarcinoma, and tumor location in the ascending, transverse, descending or sigmoid colon. Patient exclusion criteria were as follows: i) Age, $<18$ years; ii) contraindications for surgery; iii) hereditary CRC syndromes; iv) multiple primary neoplasms; v) loss of follow-up; and vi) incomplete clinical records.

A total of 114 patients were included in this study. Cancerous colon tissues and adjacent non-cancerous tissues were collected and stored in liquid nitrogen until further analysis. The clinicopathological features were obtained from medical records based on the CRC database, including age, sex, body mass index (BMI), gross type, histological differentiation, pathological TNM staging (14) and survival outcome. The variables of colon cancer are presented in Table I. Survival outcomes were obtained from the post-operative surveillance.

Patient follow-up. Post-operative surveillance was conducted four times in the first 3 years, then twice for the next 2 years and annually thereafter. Follow-up items included a physical examination, serum carcinoembryonic antigen (CEA) test, chest X-ray or CT scans, abdominopelvic MRI or CT scans, and an annual colonoscopy. The last follow-up time was recorded as the mortality of the patient or the cutoff date of September 30, 2018.

Immunohistochemistry (IHC). Immunohistochemical staining was performed using the streptavidin-biotin complex method (15), in order to determine SPRR1A protein expression. For immunohistochemical studies, surgical specimens were harvested and fixed in $10 \%$ formalin overnight at room temperature, prior to being embedded in paraffin at room temperature. Paraffin-embedded tissue samples were cut into 4- $\mu$ m-thick sections. In brief, the slides were blocked with $5 \%$ normal goat serum (Fuzhou Maixin Biotech Co., Ltd.) for $20 \mathrm{~min}$ at room temperature. Tissue sections were incubated with primary antibody directed against SPRR1A (1:200; cat. no. bs-11162R; BIOSS) overnight at $4^{\circ} \mathrm{C}$. After $24 \mathrm{~h}$, membranes were incubated with horseradish peroxidase-labeled secondary antibody (1:500; cat. no. SP KIT-C1; Fuzhou Maixin Biotech Co., Ltd.) for $1 \mathrm{~h}$ at room temperature. The slides were subsequently stained with 3,3'-diaminobenzidine for $30 \mathrm{~min}$ at room temperature and observed under an optical light microscope (magnification, x100). PBS was used as a negative control.

Tissue sections were observed and blindly scored by two independent pathologists from the Department of Pathology, Fujian Medical University Union Hospital (Fuzhou, China). The percentage of positive cells and the color were determined based on the intensity score (16). The staining intensity was scored as follows: 0 , no staining; 1 , light yellow; 2 , brown and 3 , deep brown. The percentage of positive cells was scored as follows: $0,<5 \% ; 1(5-\leq 25 \%) ; 2(>25-50 \%) ; 3,(51-75 \%)$ and 4 $(>75 \%)$. The final score was obtained by multiplying these two scores: 0 , negative (-); 1-4, weakly positive $(+) ; 5-8$, positive $(++)$ and $9-12$, strongly positive $(+++)(16)$. SPRR1A expression was divided into two groups as follows: High SPRR1A expression, final score 5-12; and low SPRR1A expression, final score $0-4$.
Bioinformatic analysis of SPRR1A. Meta-analysis of SPRR1A expression in colon cancer tissues was performed using the Oncomine database (https://www.oncomine.org). The prognostic impact of SPRR1A on CRC was evaluated using the R2: Genomics Analysis and Visualization Platform (17).

Statistical analysis. Statistical analyses were conducted using SPSS (version 24.0; IBM, Corp.). Data were presented as the mean \pm standard deviation (SD), as appropriate. Clinicopathological characteristics between groups were compared using the $\chi^{2}$, Student's t-test and paired Student's t-test, when appropriate. Survival outcomes were evaluated with the Kaplan-Meier method and compared via the log-rank test. Univariate and multivariate analysis were conducted by using a Cox regression model to evaluate the prognostic value of SPRR1A expression. $\mathrm{P}<0.05$ was considered to indicate a statistically significant difference.

\section{Results}

Baseline characteristics. A total of 114 patients with colon cancer (76 men and 38 women) were included in the present study. The mean age was 62.6 years (range, 49.2-76.1 years). Baseline clinicopathological characteristics are presented in Table I. SPRR1A expression significantly increased in colon cancer tissues compared with adjacent non-cancerous tissues via paired sample t-test analysis $(\mathrm{P}<0.001$; Fig. 1). Based on the IHC results of SPRR1, 32 patients with a final score of 0-4 were classified in the low SPRR1A expression group (Fig. 2A and B), and 82 patients with a final score of 5-12 were classified in the high SPRR1A expression group (Fig. 2C and D).

Association of SPRR1A expression with clinicopathological characteristics of patients with colon cancer. The present study subsequently evaluated the association of SPRR1A expression with the clinicopathological features patients with colon cancer (Table II). High SPRR1A expression was significantly associated with lymph node invasion $(\mathrm{P}=0.042)$; however, no significant associations were demonstrated between SPRR1A expression and other clinicopathological features, including age, sex, BMI, pretreatment CEA and carbohydrate antigen 19-9 (CA19-9) levels, tumor location, gross type of tumor, tumor histopathology, tumor differentiation and pathological $\mathrm{T}$ stage (all $\mathrm{P}>0.05$ ). The incidence of distant metastasis was $15.9 \%$ in the high SPRR1A expression group and $3.1 \%$ in the low SPRR1A expression group; however, no significant differences were observed between the two groups.

High SPRRIA expression is associated with poor survival in colon cancer. The Kaplan-Meier curve demonstrated that high SPRR1A expression was significantly associated with a worse overall survival (OS) $(\mathrm{P}=0.013$; Fig. 3A) and disease-free survival (DFS) $(\mathrm{P}=0.0 .004$, Fig. 3B) rates. The 5-year OS rates were significantly lower in patients with high SPRR1A expression levels than that of patients with low SPRR1A expression levels (64.6\% vs. 87.5\%; Fig. 3A). The 5-year DFS rate was significantly lower in patients with high SPRR1A expression levels than that of patients with low SPRR1A expression levels (66.4\% vs. $93.8 \%$; Fig. 3B). 
Table I. Patient characteristics $(\mathrm{n}=114)$.

\begin{tabular}{|c|c|}
\hline Characteristics & P-value \\
\hline Mean age $\pm S D$, years & $62.6 \pm 13.4$ \\
\hline $\mathrm{BMI}\left(\right.$ mean $\left.\pm \mathrm{SD}, \mathrm{kg} / \mathrm{m}^{2}\right)$ & $21.9 \pm 2.8$ \\
\hline \multicolumn{2}{|l|}{ Sex, n $(\%)$} \\
\hline Male & $76(66.7)$ \\
\hline Female & $38(33.3)$ \\
\hline \multicolumn{2}{|l|}{ Pretreatment CEA level, n (\%) } \\
\hline$<5 \mathrm{ng} / \mathrm{ml}$ & $68(59.6)$ \\
\hline$\geq 5 \mathrm{ng} / \mathrm{ml}$ & $46(40.4)$ \\
\hline \multicolumn{2}{|l|}{ Pretreatment CA19-9 level, n (\%) } \\
\hline$<37 \mathrm{U} / \mathrm{ml}$ & $98(86.0)$ \\
\hline$\geq 37 \mathrm{U} / \mathrm{ml}$ & $16(14.0)$ \\
\hline \multicolumn{2}{|l|}{ Tumor location, n (\%) } \\
\hline Ascending colon & $34(29.8)$ \\
\hline Transverse colon & $4(3.5)$ \\
\hline Descending colon & $11(9.6)$ \\
\hline Sigmoid colon & $65(57.0)$ \\
\hline \multicolumn{2}{|l|}{ Gross type, n (\%) } \\
\hline Expanding & $45(39.5)$ \\
\hline Ulcerated & $64(56.1)$ \\
\hline Infiltrating & $5(4.4)$ \\
\hline \multicolumn{2}{|l|}{ Histopathology, n (\%) } \\
\hline Adenocarcinoma & $101(88.6)$ \\
\hline Mucinous or signet ring adenocarcinoma & $13(11.4)$ \\
\hline \multicolumn{2}{|l|}{ Tumor differentiation, $\mathrm{n}(\%)$} \\
\hline Grade $1+2$ & $87(76.3)$ \\
\hline Grade $3+4$ & $27(23.7)$ \\
\hline \multicolumn{2}{|l|}{ T stage, $\mathrm{n}(\%)$} \\
\hline $\mathrm{T} 1$ & $7(6.1)$ \\
\hline $\mathrm{T} 2$ & $9(7.9)$ \\
\hline $\mathrm{T} 3$ & $61(53.5)$ \\
\hline $\mathrm{T} 4$ & $37(32.5)$ \\
\hline \multicolumn{2}{|l|}{ Lymph node invasion, n (\%) } \\
\hline No & $61(53.5)$ \\
\hline Yes & $53(46.5)$ \\
\hline \multicolumn{2}{|l|}{ Pathological TNM stage, n (\%) } \\
\hline I & $13(11.4)$ \\
\hline II & $44(38.6)$ \\
\hline III & $44(38.6)$ \\
\hline IV & $13(11.4)$ \\
\hline \multicolumn{2}{|l|}{ SPRR1A expression, $\mathrm{n}(\%)$} \\
\hline High & $82(71.9)$ \\
\hline Low & $32(28.1)$ \\
\hline Mean OS time \pm SD, months & $76.5 \pm 32.8$ \\
\hline
\end{tabular}

SD, standard deviation; BMI, body mass index; CEA, carcinoembryonic antigen; CA19-9, carbohydrate antigen 19-9; SPRR1A, small proline-rich 1A; OS, overall survival; TNM, Tumor-Node-Metastasis.

High SPRRIA expression is a prognostic indicator in patients with colon cancer. Univariate analysis identified 10 potential

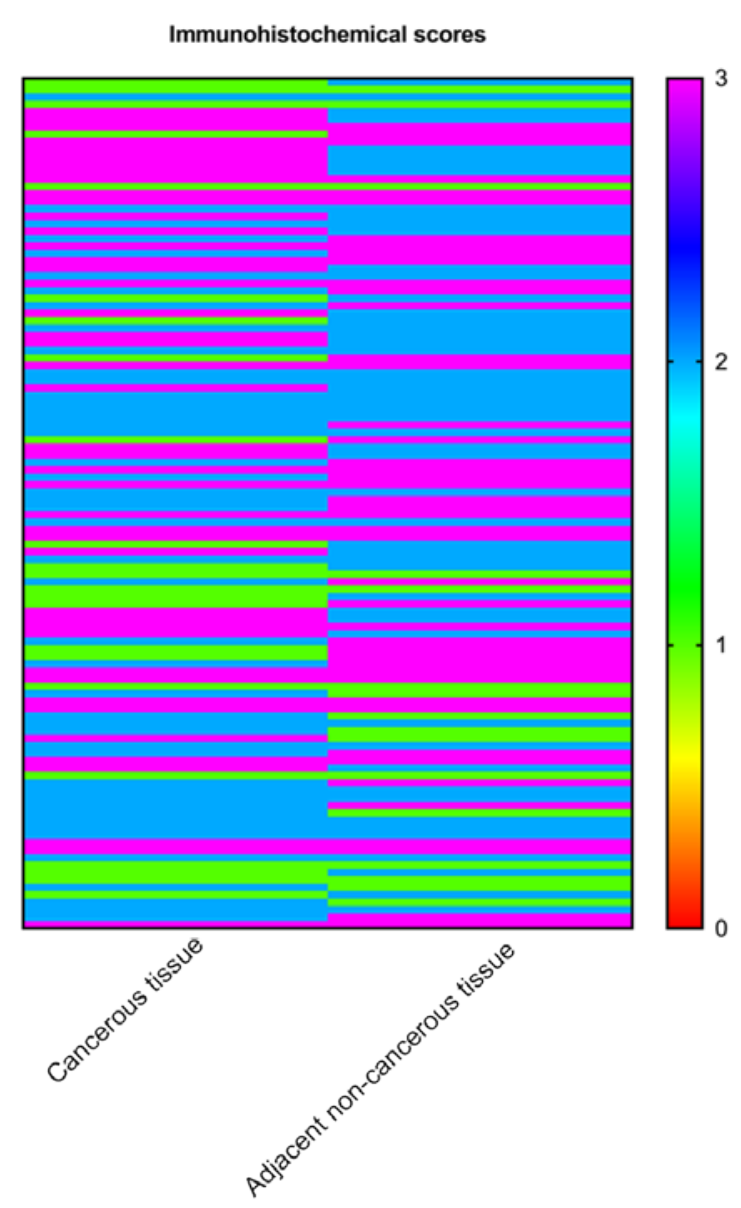

Figure 1. Heatmap of the immunohistochemical score of SPRR1A in cancerous and adjacent noncancerous colon tissues. Every unit represents the SPRR1A expression in each patient. SPRR1A, small proline-rich protein 1A.

risk factors associated with OS, including pathological T stage [hazard ratio (HR), 2.669; $\mathrm{P}<0.001$ ], pathological $\mathrm{N}$ stage $(\mathrm{HR}$, 4.323; $\mathrm{P}<0.001)$ and high SPRR1A expression (HR, 3.083; $\mathrm{P}=0.019$ ). In addition, it was indicated that age, sex, BMI, histology, differentiation, pretreatment CEA and CA19-9 levels were not significantly associated with OS. Cox regression analysis demonstrated that pathological $\mathrm{T}$ stage (HR, 2.099; $\mathrm{P}=0.012)$, pathological $\mathrm{N}$ stage $(\mathrm{HR}, 2.832 ; \mathrm{P}=0.007)$ and high SPRR1A expression (HR, 2.606; $\mathrm{P}=0.047)$ remained independent prognostic factors for OS, as presented in Table III.

Validation of SPRRIA in Oncomine and R2. SPRR1A expression was verified, along with its prognostic value via bioinformatics analysis. Meta-analysis of $21 \mathrm{GEO}$-sourced datasets from the Oncomine database was performed and the results revealed that SPRR1A mRNA levels were significantly increased in $\mathrm{CRC}$ tissues $(\mathrm{P}<0.001$; Fig. 4A). The R2 platform was used to generate Kaplan-Meier OS curves using the following datasets: 'Tumor Colon-Sieber-290-MAS5.0-u 133p2', 'Tumor Colon-Smith-232-MAS5.0-u133p2', 'Tumor Colon (Core-Exon)-Sveen-333-rma_sketch-huex10p', 'Tumor Colon (Core-Transcript)-Sveen-333-rma_sketch-huex10t', 'Tumor Colon CIT (Combat)-Marisa-566-rma-u133p2', 'Tumor Colon MSI-status (Core Exon)-Sveen-95-rma sketch-huex10p' and 'Tumor Colon MVRM-SieberSmith-345 
A

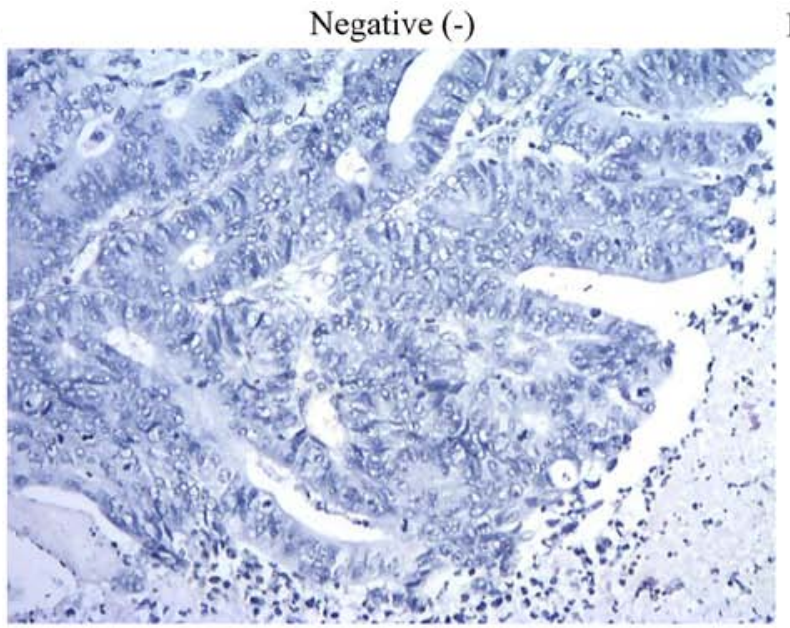

$\mathrm{C}$

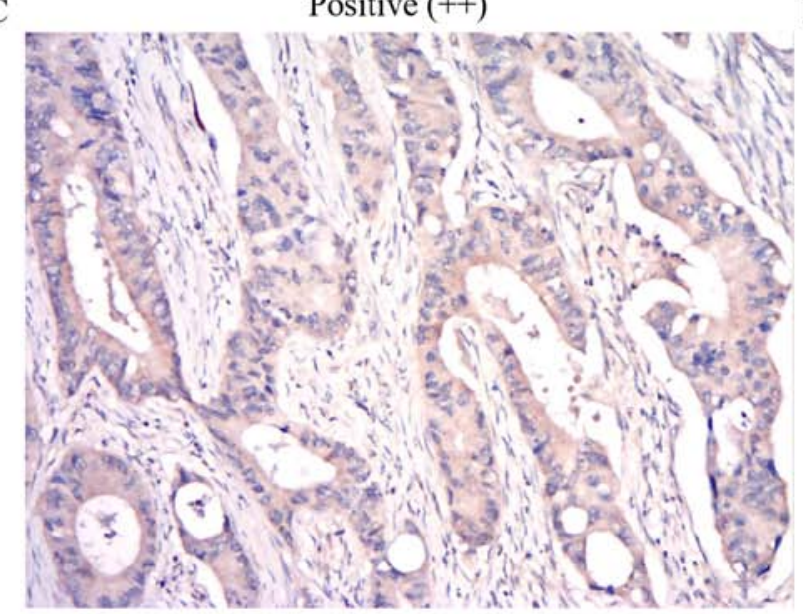

B

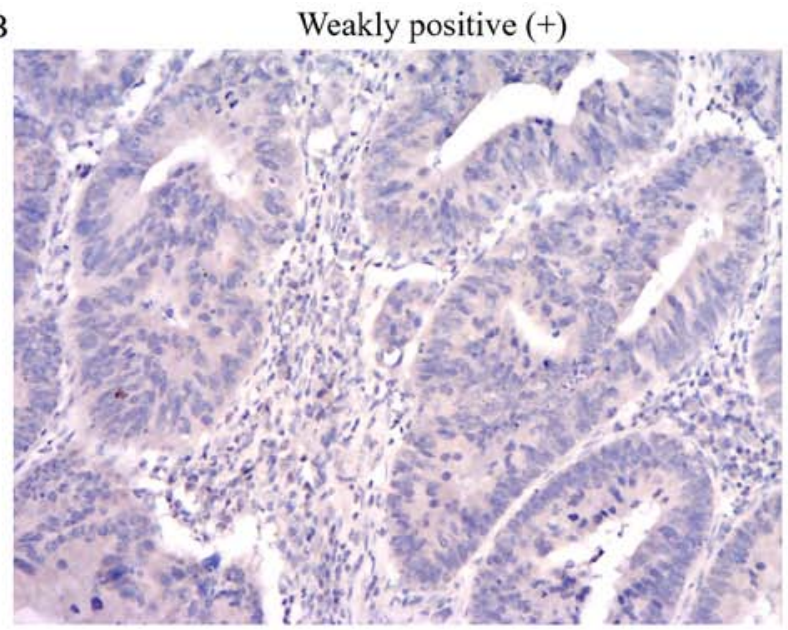

$\mathrm{D}$

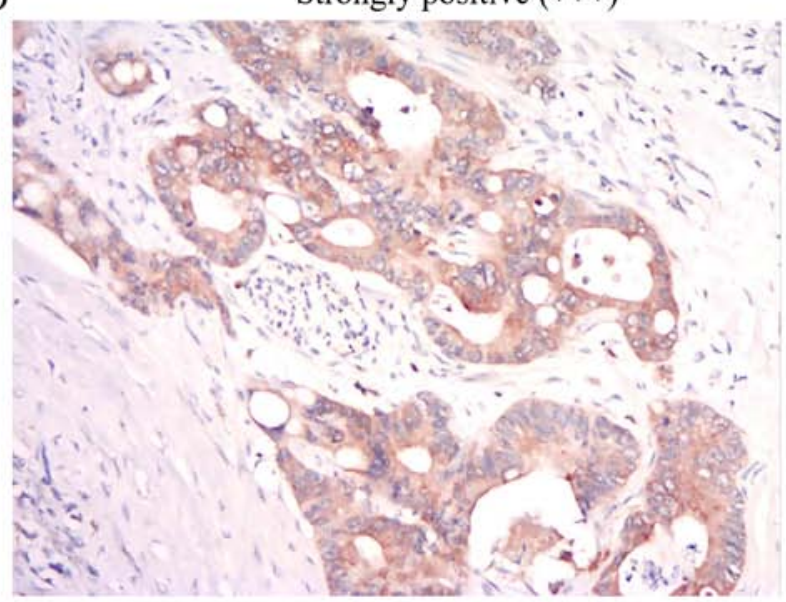

Figure 2. Representative images of small proline-rich protein 1A expression in colon cancer tissues (magnification, x100). (A) Negative (-), (B) weakly positive $(+),(\mathrm{C})$ positive $(++)$ and (D) strongly positive $(+++)$.
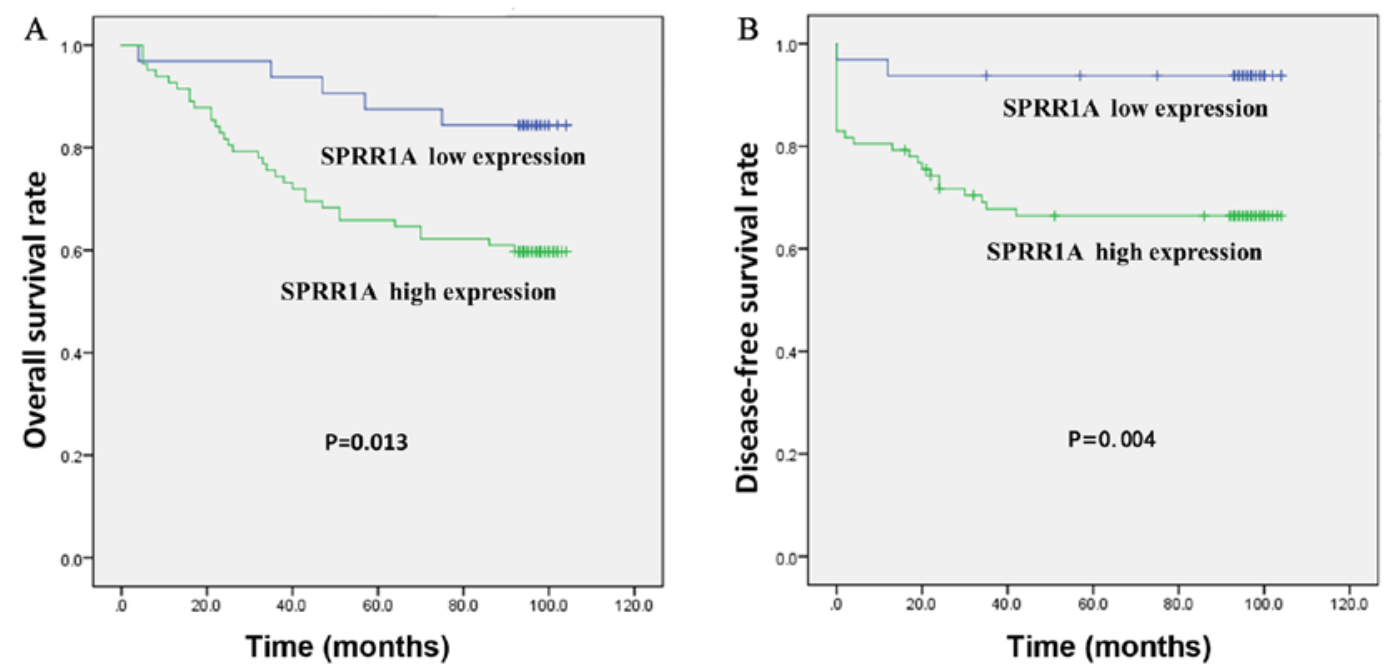

Figure 3. Kaplan-Meier curves for patients with colon cancer stratified by SPRR1A expression level. (A) Overall survival and (B) disease-free survival rates for patients with colon cancer. SPRR1A, small proline-rich protein 1A.

-fRMA(bc)-u133p2' (17). High SPRR1A expression was associated with significantly worse rates of event- and relapse-free survival rate (all $\mathrm{P}<0.05$; Fig. $4 \mathrm{~B}-\mathrm{H}$ ). In the 'Tumor Colon (KRAS mut)-Hase-59-MAS5.0-u133p2' (P=0.064; Fig. 4I) and
'Tumor Colon MSI-status (Core-Transcript)-Sveen-95-rma sketch-huex10t' ( $\mathrm{P}=0.259$; Fig. 4J) data sets, high SPRR1A expression was associated with a worse OS; however, no statistically significant differences were observed. 
Table II. Association between SPRR1A expression and clinicopathological characteristics in patients with colon cancer.

\begin{tabular}{|c|c|c|c|}
\hline \multirow[b]{2}{*}{ Characteristics } & \multicolumn{2}{|c|}{ SPRR1A expression } & \multirow[b]{2}{*}{ P-value } \\
\hline & Low $(n=32)$ & $\operatorname{High}(\mathrm{n}=82)$ & \\
\hline Mean age $\pm S D$, years & $64.0 \pm 12.4$ & $62.1 \pm 13.8$ & 0.685 \\
\hline Mean $\mathrm{BMI} \pm \mathrm{SD}, \mathrm{kg} / \mathrm{m}^{2}$ & $21.5 \pm 2.8$ & $22.1 \pm 2.8$ & 0.683 \\
\hline Pretreatment CEA, n (\%) & & & 0.525 \\
\hline$<5 \mathrm{ng} / \mathrm{ml}$ & $21(65.6)$ & $47(57.3)$ & \\
\hline$\geq 5 \mathrm{ng} / \mathrm{ml}$ & $11(34.4)$ & $35(42.7)$ & \\
\hline Pretreatment CA19-9, n (\%) & & & 0.760 \\
\hline$<37 \mathrm{ng} / \mathrm{ml}$ & $27(84.4)$ & $71(86.6)$ & \\
\hline$\geq 37 \mathrm{ng} / \mathrm{ml}$ & $5(15.6)$ & $11(13.4)$ & \\
\hline Pathological T stage, n (\%) & & & 0.337 \\
\hline $\mathrm{T} 1$ & $4(12.5)$ & $3(6.1)$ & \\
\hline $\mathrm{T} 2$ & $3(9.4)$ & $6(7.9)$ & \\
\hline $\mathrm{T} 3$ & $17(53.1)$ & $50(61.0)$ & \\
\hline $\mathrm{T} 4$ & $8(25.0)$ & $23(28.0)$ & \\
\hline Lymph node invasion, n (\%) & & & 0.042 \\
\hline No & $22(68.8)$ & $39(47.6)$ & \\
\hline Yes & $10(31.3)$ & $43(52.4)$ & \\
\hline Distant metastasis, n (\%) & & & 0.108 \\
\hline No & $31(96.9)$ & $69(84.1)$ & \\
\hline Yes & $1(3.1)$ & $13(15.9)$ & \\
\hline Tumor location, n (\%) & & & 0.444 \\
\hline Ascending colon & $13(40.6)$ & $21(25.7)$ & \\
\hline Transverse colon & $1(3.1)$ & $3(3.6)$ & \\
\hline Descending colon & $2(6.3)$ & $9(10.9)$ & \\
\hline Sigmoid colon & $16(50.0)$ & $49(59.8)$ & \\
\hline Tumor differentiation, n (\%) & & & 0.836 \\
\hline Grade $1+2$ & $24(75.0)$ & $63(76.8)$ & \\
\hline Grade $3+4$ & $8(25.0)$ & $19(23.2)$ & \\
\hline
\end{tabular}

SD, standard deviation; BMI, body mass index; CEA, carcinoembryonic antigen; CA19-9, carbohydrate antigen 19-9; SPRR1A, small proline-rich $1 \mathrm{~A}$.

Table III. Univariate and multivariate analyses of risk factors for overall survival of patients with colon cancer.

\begin{tabular}{|c|c|c|c|c|}
\hline \multirow[b]{2}{*}{ Variables } & \multicolumn{2}{|c|}{ Univariate analysis } & \multicolumn{2}{|c|}{ Multivariate analysis } \\
\hline & $\operatorname{HR}(95 \% \mathrm{CI})$ & P-value & $\mathrm{HR}(95 \% \mathrm{CI})$ & P-value \\
\hline Age (years) & $1.021(0.994-1.048)$ & 0.136 & & \\
\hline Sex (female vs. male) & $1.818(0.860-3.842)$ & 0.117 & & \\
\hline BMI & $1.064(0.955-1.187)$ & 0.261 & & \\
\hline Pretreatment CEA level ( $<5 \mathrm{vs} . \geq 5 \mathrm{ng} / \mathrm{ml})$ & $0.611(0.323-1.155)$ & 0.129 & & \\
\hline Pretreatment CA19-9 level (<37 vs. $\geq 37 \mathrm{U} / \mathrm{ml}$ ) & $0.876(0.366-2.096)$ & 0.766 & & \\
\hline Pathological T stage & $2.669(1.551-4.594)$ & $<0.001$ & $2.099(1.175-3.749)$ & 0.012 \\
\hline Pathological N stage & $4.323(2.093-8.928)$ & $<0.001$ & $2.832(1.335-6.008)$ & 0.007 \\
\hline Histopathology (adenocarcinoma vs. mucinous adenocarcinoma) & $1.745(0.784-3.886)$ & 0.173 & & \\
\hline Tumor differentiation (grade $1+2$ vs. $3+4$ ) & $0.755(0.367-1.554)$ & 0.445 & & \\
\hline SPRR1A expression (high vs. low) & $3.083(1.203-7.903)$ & 0.019 & $2.606(1.012-6.708)$ & 0.047 \\
\hline
\end{tabular}

HR, hazard ratio; CI, confidence interval; BMI, body mass index; CEA, carcinoembryonic antigen; CA19-9, carbohydrate antigen 19-9; SPRR1A, small proline-rich 1A. 

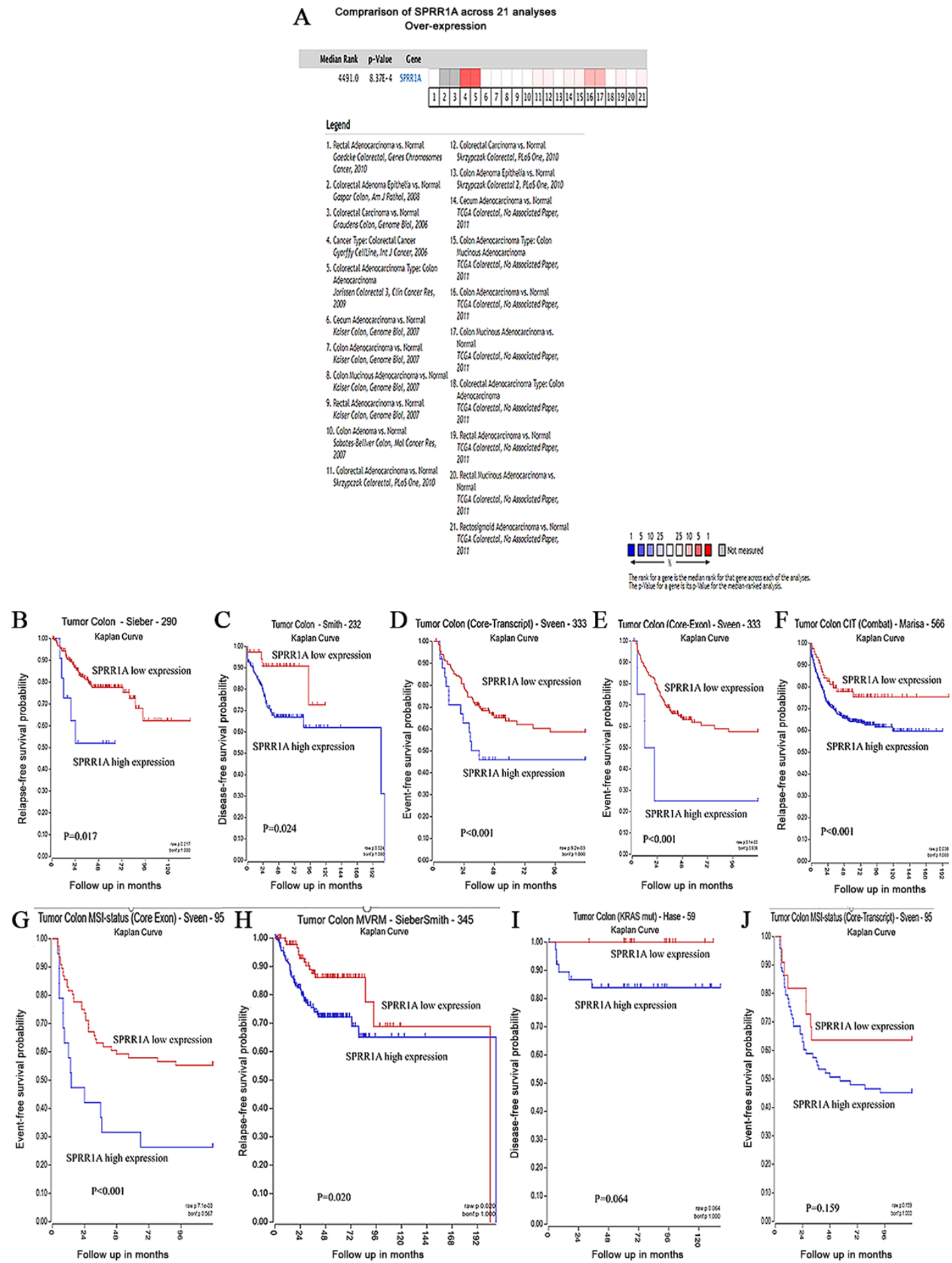

Figure 4. Verification of SPRR1A expression. (A) Meta-analysis of 21 GEO-sourced datasets mined from the Oncomine database demonstrated that SPRR1A mRNA levels were significantly lower in colorectal cancer tissues than in normal colon tissues $(\mathrm{P}<0.001)$. (B-H) High expression of SPRR1A was associated with significantly poorer event- and relapse-free survival times (All $\mathrm{P}<0.05$ ). (I and J) High expression of SPRR1A was associated with poorer event- and relapse-free survival; however, no significant differences were observed ( $\mathrm{P}=0.141$ and $\mathrm{P}=0.118$ ). SPRR1A, small proline-rich protein $1 \mathrm{~A}$. Event-free survival indicated that the patient was still alive. Relapse-free and disease-free survival indicated no tumor recurrence in patients.

\section{Discussion}

Few studies have focused on the association between SPRR1A expression and the prognosis of colon cancer $(9,11,12)$. Through use of immunohistochemical analysis, the present study demonstrated that SPRR1A expression is increased in colon cancer tissues compared with adjacent noncancerous tissues, which was significantly associated with lymph node involvement. In addition, high SPRR1A expression was an independent predictor for OS. Using bioinformatics analysis, the association between higher SPRR1A expression and impaired prognosis was further confirmed.

Previous studies have demonstrated that SPRR1A plays a crucial role in the pathogenesis of various types of cancer, 
such as diffuse large B-cell lymphomas (9), head and neck squamous cell carcinoma (10) and breast (12) cancer. Leclerc et al (12) reported high expression of SPRR1A in a murine preneoplastic intestine and in the normal intestinal mucosa of patients with CRC. In addition, only a limited number of mice with high expression of SPRR1A protein eventually develop tumors (12). The present study showed that SPRR1A expression was significantly higher in cancerous tissues. However, the underlying mechanism remains unclear. Given that colonic carcinogenesis is a multistage process involving environmental and genetic changes (13), one of the possible explanations may be that the normal intestinal mucosa in patients with colon cancer has sufficient time for compensatory expression changes. Therefore, the present study investigated whether the high expression of SPRR1A serves as an oncogene in colon cancer progression. To the best of our knowledge, this study provided novel insight demonstrating that the high expression of SPRR1A is associated with lymph node metastasis, suggesting the clinical importance of SPRR1A in colon cancer.

The prognostic significance of SPRR1A has been demonstrated in different types of cancer including, diffuse large B-cell lymphomas, epithelial-like head and neck carcinoma and progesterone receptor-positive breast cancer (9-11). However, the clinical impact of SPRR1A as a prognostic biomarker for colon cancer remains unclear. In the present study, the prognostic significance of SPRR1A expression was further examined in colon cancer. It was revealed that high SPRR1A expression was associated with significantly worse OS and DFS rates. After adjusting for confounding factors, it was demonstrated that the expression of SPRR1A was an independent predictor for OS. In accordance with previous findings $(10,11)$, these results revealed the prognostic implication of SPRR1A in colon cancer. A tendency was indicated between high SPRR1A expression and the incidence of distant metastasis (high expression vs. low expression, 15.9\% vs. 3\%); however, no significant differences were indicated. In addition, this study was not able to identify high SPRR1A expression as an independent predictor for tumor recurrence. The small patient sample size of this study cohort could contribute to the lack of statistical difference. Given the complex mechanism of tumor recurrence and metastasis, large-scale studies are required to investigate the underlying mechanism.

Furthermore, the association between SPRR1A expression and survival in colon cancer was evaluated using bioinformatics analysis. The results demonstrated significantly higher SPRR1A mRNA levels in CRC tissues, and high SPRR1A expression was associated with a worse survival time. Collectively, these findings were consistent with the aforementioned results.

In summary, SPRR1A expression was upregulated in colon cancer tissues and high SPRR1A expression was associated with lymph node metastasis and impaired survival in patients with colon cancer. The results suggest that SPRR1A may act as a potential biomarker for the prognosis of colon cancer.

\section{Acknowledgements}

Not applicable.

\section{Funding}

The present study was funded by the National Natural Science Foundation of China (grant no. 81472777), the Foundation of Science and Technology Innovation Project in Fujian Province (grant no. 2016Y9026), the Training Plan of Middle-Aged and Science Foundation of the Fujian Province (grant no. 2017J01296) and the National Clinical Key Specialty Construction Project (General Surgery) of China (grant no. 2012-649).

\section{Availability of data and materials}

The datasets used and/or analyzed during the present study are available from the corresponding author upon reasonable request.

\section{Authors' contributions}

YD, XZ, YYZ, XRL, and ZBX designed the study, performed the experiments, interpreted the data and drafted the initial manuscript. XZ and MFX observed and blindly scored IHC. JP, CWY, PC and MXL provided the tissue specimens and interpreted the data. All authors read and approved the final manuscript.

\section{Ethics approval and consent to participate}

The present study was ethically approved by the Institutional Review Board of Fujian Medical University Union Hospital. Written informed consent was obtained from all patients prior to the study start.

\section{Patient consent for publication}

Not applicable.

\section{Competing interests}

The authors declare that they have no competing interests.

\section{References}

1. Bray F, Ferlay J, Soerjomataram I, Siegel RL, Torre LA and Jemal A: Global cancer statistics 2018: GLOBOCAN estimates of incidence and mortality worldwide for 36 cancers in 185 countries. CA Cancer J Clin 68: 394-424, 2018.

2. Mahar AL, Compton C, Halabi S, Hess KR, Weiser MR and Groome PA: Personalizing prognosis in colorectal cancer: A systematic review of the quality and nature of clinical prognostic tools for survival outcomes. J Surg Oncol 116: 969-982, 2017.

3. Bardhan K and Liu K: Epigenetics and colorectal cancer pathogenesis. Cancers (Basel) 5: 676-713, 2013.

4. Mouradov D, Domingo E, Gibbs P, Jorissen RN, Li S, Soo P, Lipton L, Desai J, Danielsen HE, Oukrif D, et al: Survival in stage II/III colorectal cancer is independently predicted by chromosomal and microsatellite instability, but not by specific driver mutations. Am J Gastroenterol 108: 1785-1793, 2013.

5. Das V, Kalita J and Pal M: Predictive and prognostic biomarkers in colorectal cancer: A systematic review of recent advances and challenges. Biomed Pharmacother 87: 8-19, 2017.

6. Fujimoto W, Nakanishi G, Arata J and Jetten AM: Differential expression of human cornifin alpha and beta in squamous differentiating epithelial tissues and several skin lesions. J Invest Dermatol 108: 200-204, 1997. 
7. Gibbs S, Fijneman R, Wiegant J, van Kessel AG, van De Putte P and Backendorf C: Molecular characterization and evolution of the SPRR family of keratinocyte differentiation markers encoding small proline-rich proteins. Genomics 16: 630-637, 1993.

8. De Heller-Milev M, Huber M, Panizzon R and Hohl D: Expression of small proline rich proteins in neoplastic and inflammatory skin diseases. Br J Dermatol 143: 733-740, 2000.

9. Zhang H, Gao J, Zhao Z, Li M and Liu C: Clinical implications of SPRR1A expression in diffuse large B-cell lymphomas: A prospective, observational study. BMC Cancer 14: 333, 2014.

10. Pavón MA, Arroyo-Solera I, León X, Téllez-Gabriel M, Virós D, Gallardo A, Céspedes MV, Casanova I, Lopez-Pousa A, Barnadas A, et al: The combined use of EFS, GPX2, and SPRR1A expression could distinguish favorable from poor clinical outcome among epithelial-like head and neck carcinoma subtypes. Head Neck 41: 1830-1845, 2019.

11. Chen G, Li G, Luo M, Wei X, Wang D, Zhang H, Zhao X, Chen B and Liu C: Clinical significance of SPRR1A expression in progesterone receptor-positive breast cancer. Tumour Biol 36: 2601-2605, 2015.

12. Leclerc D, Lévesque N, Cao Y, Deng L, Wu Q, Powell J, Sapienza C and Rozen R: Genes with aberrant expression in murine preneoplastic intestine show epigenetic and expression changes in normal mucosa of colon cancer patients. Cancer Prev Res (Phila) 6: 1171-1181, 2013.
13. Hammoud SS, Cairns BR and Jones DA: Epigenetic regulation of colon cancer and intestinal stem cells. Curr Opin Cell Biol 25: 177-183, 2013.

14. Edge S: American Joint Committee on Cancer: AJCC cancer staging manual. 7th edition. New York, Springer, 2010.

15. Qiu J, Liu P, Shi C and Han B: Low-grade myofibroblastic sarcomas of the maxilla. Oncol Lett 9: 619-625, 2015.

16. Zhang Y, Xu Z, Sun Y, Chi P and Lu X: Knockdown of KLK11 reverses oxaliplatin resistance by inhibiting proliferation and activating apoptosis via suppressing the PI3K/AKT signal pathway in colorectal cancer cell. Onco Targets Ther 11: 809-821, 2018.

17. Koster J, Molenaar J and Versteeg R: Abstract B1-05: R2: Accessible web-based genomics analysis and visualization platform for biomedical researchers. Cancer Res 757: B1-B5, 2015.

This work is licensed under a Creative Commons Attribution-NonCommercial-NoDerivatives 4.0 International (CC BY-NC-ND 4.0) License. 